\title{
Polarization quadrature measurement of subwavelength diffracting structures
}

\author{
D. S. Marx and D. Psaltis
}

\begin{abstract}
The amplitude and the phase of the diffracted far field depends on polarization when the diffracting structure is comparable to or less than the wavelength. When the far-field amplitude and the phase of one polarization with respect to the orthogonal polarization is measured, small changes in the structure can be measured. To make the far-field polarization measurements, we design a detector that measures the relative polarization amplitude and the phase in quadrature. We predict numerically and verify experimentally the polarization amplitude and the phase for an optical disc and a set of gratings with varying depth. Our results show that this measurement technique is sensitive to small variations in the diffracting structure and that it can be useful in applications such as critical dimension and overlay metrology in microelectronics fabrication. (C) 1997 Optical Society of America

Key words: Vector diffraction, metrology, scatterometry, optical disk memories.
\end{abstract}

\section{Introduction}

The limits to the resolution of optical imaging systems, and the effects of diffraction on such systems, are well known. As the size of the diffracting structure becomes less than the wavelength of the incident light, scalar diffraction theory no longer adequately describes the diffraction and polarization becomes an important consideration. Also, the optical measurement of such a structure becomes very imprecise because spatial information of the structure does not propagate to the far field. However, classical resolution limits are most relevant when no a priori information about the object is available.

In the case of optical systems such as optical disc memories and microelectronics measurement and inspection systems, significant information about the object is available. For example, an optical disc player is designed to read a specific format that specifies the parameters of the information pits and the track pitch. Metrology of subwavelength features by scatterometry has been demonstrated with high

When the research was done the authors were with the California Institute of Technology, Department of Electrical Engineering, Mail Code 136-93, Pasadena, California. D. S. Marx is currently with Sight Systems, Inc., Suite 119, 3541 Old Conejo Road, Newbury Park, California 91320.

Received 17 June 1996; revised manuscript received 9 December 1996.

0003-6935/97/256434-07\$10.00/0

(C) 1997 Optical Society of America resolution.1,2 These experiments measured the parameters of diffraction gratings with high resolution, and the assumption that the object was a grating provided the necessary a priori information. However, the requirement of a grating places a burden on developing a metrology system for microelectronics applications. We avoid this requirement by using a tightly focused spot formed with a high numerical aperture lens. Although this spot illuminates a single structure for measurement, information about the object is still required. The method presented here assumes that knowledge about the object, for example, a line or a trench, is available and that the measurement of its deviation from some expected object is desired.

Since the pits embossed on optical disks will be less than half of the readout wavelength for the next generation digital videodisc ${ }^{3}$ (DVD) and the minimum feature size used in microelectronic circuits is now $0.25 \mu \mathrm{m}$ and decreasing, we expect that the diffraction of light from such structures to be polarization dependent. The first purpose of the work presented here is to study and to predict the nature of the polarization effects on diffraction. The second purpose is to use the polarization effects as a novel method of measurement. This proposed measurement technique can be useful for both reducing the cost and increasing the efficiency of the metrology and the inspection of high volume, small feature-size microelectronics fabrication.

We present both numerical and experimental results. To simulate the vector diffraction resulting 
from single, subwavelength structures, we used an integral method of numerical diffraction. ${ }^{4,6}$ Each calculation described here is for two-dimensional diffraction geometries. In this case, the diffracting structures, for example, lines and grooves, have infinite length, and the two-dimensional cross section of the structure is used in the simulation. The incident field used in the simulations was a focused cylindrical spot formed by the integration of the angular spectrum over a specified numerical aperture. In this cylindrical geometry, the electromagnetic fields decompose into two independent polarizations. We define the TE polarization as the case in which the electric field is perpendicular to the plane of incidence and parallel to the line or groove, and the TM polarization is for the electric field lying in the plane of incidence.

In Section 2, we describe the polarization effects predicted by the numerical calculations for the new, recently agreed upon DVD format. ${ }^{3}$ In this example we find that an important difference in the diffraction of the two polarizations for a reflecting groove is that the TE polarization does not penetrate the groove, whereas the TM light does. This difference in the phase at the bottom of grooves for the two polarizations was previously pointed out by Dil and Jacobs, ${ }^{4}$ and by Kok and Gallagher, ${ }^{5}$ for plane-wave incidence. In Section 3 we present a quadrature detector to measure the phase difference between the TE and TM polarizations in the diffracted far field. A demonstration of this type of amplitude and phase measurement is described in Section 4 for the DVD. Finally, we demonstrate the potential of using polarization in quadrature for the measurement of subwavelength lines or grooves by measuring aluminum gratings of different depths.

\section{Simple Example: New DVD Format with Varying Pit Depth}

The improvement in storage density from CD-audio to DVD is obtained through an increase in the NA of the objective lens and a decrease in the wavelength of the source. This combination produces a smaller spot width. Further gain results from making the track pitch significantly less than the spot width. Because of these changes, the pit width becomes less than half of the wavelength. For the CD-audio format, the reflected signal caused by the diffraction of the incident light with the track is adequately described by scalar explanations, and the difference between the TE- and TM-reflected fields is small. ${ }^{7}$ However, for the DVD format, the two polarizations show significant differences in the diffracted far field because of reductions in the track pitch and pit width with respect to wavelength. To examine the dependence of the diffracted field on polarization, we consider the electric field along the surface of the pit. According to a first-order scalar approximation, the electric field at the bottom of the pit is simply phase retarded with respect to the field at the top of the pit by $2 \pi n z_{0} / \lambda \mathrm{rad}$, where $z_{0}$ is the depth of the pit, $n$ is the index of refraction of the incident medium, and $\lambda$

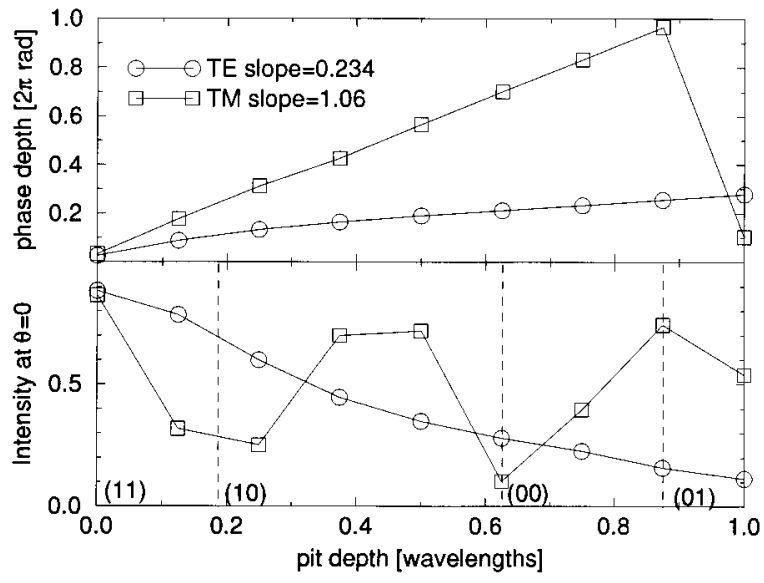

Fig. 1. Calculated pit phase depth and far-field intensity for the DVD format. The dashed vertical lines indicate which pit depths can be used to represent four logical states.

is the wavelength. However, when the pit width becomes sufficiently small, this scalar approximation is no longer accurate, and we find that the phase of the electric field at the bottom of the pit depends on the polarization of the incident light and is typically different from the scalar prediction. The apparent pit depth, or phase depth, is $z_{p}=2 \pi \phi / \lambda$, where $\phi$ is the phase of the electric field at the bottom of the pit. In Fig. 1, we show the results of numerical calculations of phase depth and far-field intensity for the DVD format. The calculations were made with an integral method that is described in Refs. 4, 6, and 7. The top graph in Fig. 1 shows the phase of the electric field at the bottom of the pit as a function of pit depth. The phase of the TM field is an accurate representation of the actual pit depth because its slope is near 1 . However, the TE field does not seem to be as strongly affected. Whatever the pit's depth, the TE field does not significantly retard. The far field is a combination of the light reflected from the bottom of the pit and the area around the pit. If the field at the bottom of the pit is retarded with respect to the fields reflected from the area around the pit, the intensity at the center of the far field will be reduced by the destructive interference. The central far-field intensity calculated for the DVD format (bottom graph of Fig. 1) has the appropriate variation corresponding to the pit phase depth. This difference in the far-field intensity variation for the different polarizations stimulates the following idea for the doubling of the information density of DVD's. Rather than each pit having two possible depths, zero or one-quarter wave as in the current DVD format, each pit can have four possible depths, and the depths can be distinguished by the relative far-field response of the TE and TM polarizations. The dashed vertical lines in the farfield plot of Fig. 1 indicate pit depths where four states are distinguishable. Placing a polarizing beam splitter in the return path allows independent measurement of the reflection of each polarization, thus doubling the information density of each pit.

Why should the TE polarization behave so differ- 


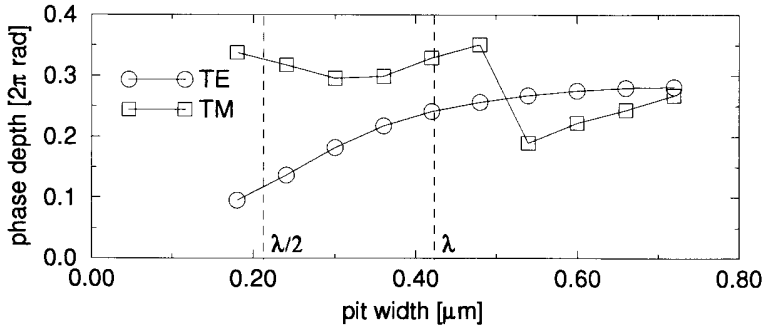

Fig. 2. Pit phase depth for different pit widths with the pit depth equal to a quarter wave. In the region where the pit width is between $\lambda / 2$ ( $\lambda$ is adjusted for the index of refraction of the incident medium) and $\lambda$, the phase depth for TE illumination varies almost a quarter wave, but it remains fairly constant for TM illumination.

ently for the CD-audio and DVD formats? The biggest change between the two formats, from the point of view of calculating diffraction, seems to be the pit width in relation to the wavelength. For both formats, the pit width is approximately one third $(\lambda /$ $N A$ ), where $\lambda$ is the wavelength, and $N A$ is the numerical aperture of the focusing objective. However, the numerical aperture increases by one-third for the DVD format, thereby reducing the pit width in relation to the wavelength. To study the effect of changing the pit width, we calculated the pit phase depth for the DVD format as a function of pit width (see Fig. 2). In the region where the pit width varies between $\lambda / 2$ and $\lambda$ (here $\lambda$ is the wavelength in the polycarbonate medium), the phase depth of the TM wave remains fairly constant while the phase depth of the TE wave increases close to the quarter-wave depth of the pit. Therefore the pit width is crucial to the penetration of the TE wave into the pit. The sudden change in phase depth for the TM curve at the pit width $\approx 0.5 \mu \mathrm{m}$ is due to the edge of the pit in the neighboring track appearing under the illumination spot.

We can explain the difference between the TE and TM dependence on the pit width by considering the case of a plane, parallel waveguide with perfectly conducting walls. The fields in this waveguide consist of discrete modes, as restricted by the boundary conditions of the tangential electric field at the walls. The TE polarization for the pit corresponds to the electric field parallel to the walls of the waveguide, and the solutions for the electric field inside the waveguide are

$$
E_{(x, z)}=\sin (p \pi x / d) \exp \left(j k_{z} z\right), k_{z}=\left[k^{2}-\left(\frac{p \pi}{d}\right)^{2}\right]^{1 / 2},
$$

where $d$ is the width of the waveguide $(k=2 \pi)$ and $p$ is an integer. When the magnetic field is parallel to the walls, the solutions are

$$
H_{(x, z)}=\cos (p \pi x / d) \exp \left(j k_{z} z\right), k_{z}=\left[k^{2}-\left(\frac{p \pi}{d}\right)^{2}\right]^{1 / 2} \text {. }
$$

Each mode, corresponding to an integer value of $p$, has a cutoff width, $d_{c}^{p}=p \pi / k$. For widths smaller than $d_{c}^{p}, k_{z}$ is imaginary, and the field amplitude weakens exponentially in $z$. For the mode $p=0$, the $\mathrm{TE}$ solution is $E_{y}=0$; there can be no TE fields. However, there is a TM solution for $p=0: H_{y}=$ $\exp (j k z)$. The cutoff width for the $p=1$ mode is $d_{c}{ }^{1}$ $=\lambda / 2$, and both TE and TM have propagating solutions for this mode when $d>d_{c}{ }^{1}$. Therefore, when $d<\lambda / 2$, only evanescent TE waves can exist although the TM zero mode can still propagate. The phase retardation of the evanescent TE wave has previously been discussed regarding the formation of binary, metallic gratings of subwavelength grooves with an effective index. ${ }^{8}$ Here lies the essential difference between the TE and the TM fields in our pit width calculations described above. When the pit width falls below half of a wavelength, the TE fields can no longer penetrate the pit, although the TM fields can still propagate to the bottom and back. The basic principle, which we will exploit with our proposed measurement technique, is that when the pit width is small, we can have pits that the TM field sees and the TE field does not. Thus, as diffracting structures become small, a new measurement technique can be used that is very sensitive to small changes in the structure's dimensions and is completely independent of the classical imaging resolution limits.

\section{Polarization Quadrature for Measuring Phase Difference}

Now that we know how the amplitude and the phase of the TM far field are modulated with respect to the TE far field, we need to develop a method of detecting this modulation. In this section we explain our proposal for detecting the TM field in quadrature by using the TE field as a reference. This detection scheme has an advantage over interferometric detection because of the ease with which both the real (inphase) and imaginary (quadrature phase) parts of the TM field can be detected. This type of detection is analogous to the detection of quadrature amplitude modulation in time domain communications systems. We display the results of our simulations and experiments as signal constellations, following the analogy to quadrature amplitude modulation constellations.

Normally, the phase of an optical field cannot be detected without interference by a reference optical field. In polarization quadrature measurement (PQM), we will illuminate the diffracting structure with both TE- and TM-polarized fields. Because the TM field is more strongly affected by the diffraction than the TE field, we can use the TE field as a reference to analyze the phase and the amplitude change in the TM field. Measuring the relationship between the two orthogonally polarized fields is similar to the conoscopic principle. ${ }^{9,10}$ The advantage of using the TE field as a reference, rather than constructing a traditional interferometer by splitting off part of the TM field before the diffraction and the interference of the two parts, is that coherence length, stability, and vibrations are not an issue. Another advantage is the ease of measuring both the inphase 


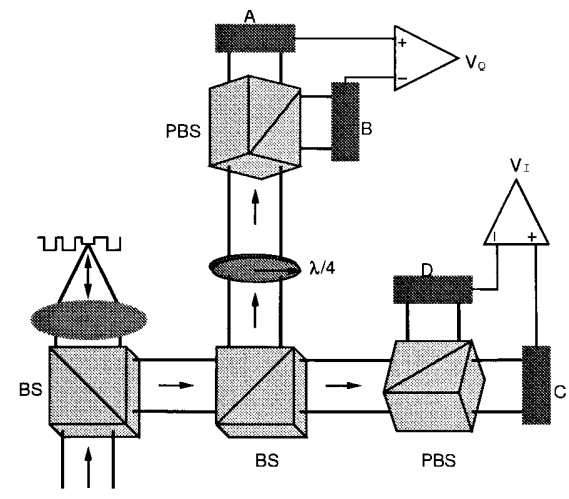

Fig. 3. Inphase and quadrature detection of the TM far field. The TE field was used as a reference.

and quadrature components. To measure both inphase and quadrature components of an electromagnetic field with traditional interferometry requires a separate reference leg with an optical path-length one-quarter wave different from the first reference leg.

The electric fields and corresponding detector voltages used in the following formulation can be considered as functions of the pupil plane of the objective. In addition, we will use the convention that the TEreflected field is polarized in the $x$ direction, so that the TM electric field points in the $y$ direction and propagation is in the $z$ direction. Then the total electric far field reflected from the sample is

$$
\mathbf{E}_{1}=a_{e} \exp \left(j \phi_{e}\right) \hat{x}+a_{m} \exp \left(j \phi_{m}\right) \hat{y},
$$

where $a_{e}, \phi_{e}$ are the amplitude and the phase of the reflected TE field, $a_{m}, \phi_{m}$ are for the TM field, and we have ignored the factor of $\exp [j(k z-\omega t)]$. The quadrature detector illustrated in Fig. 3 comprises four detectors. The inphase leg consists of a polarizing beam splitter rotated $45^{\circ}$ with respect to the $x$ axis. This rotated analyzer interferes the TE- and the TM-polarized waves. The quadrature leg is the same as the inphase leg except for the addition of a quarter-wave plate that retards the TE component by one-quarter wave with respect to the TM component. The electric field in this leg is

$$
\mathbf{E}_{2}=a_{e} \exp \left[j\left(\phi_{e}+\pi / 2\right)\right] \hat{x}+a_{m} \exp \left[j \phi_{m}\right] \hat{y} .
$$

In both legs, the signal bias due to measuring intensity is removed by subtracting the complementary outputs of the polarizing beam splitters. The voltages at detectors $\mathrm{A}$ and $\mathrm{C}$ are

$$
V_{\mathrm{A}, \mathrm{C}} \propto \frac{1}{2}\left|\mathbf{E}_{1,2} \cdot(\hat{x}+\hat{y})\right|^{2}
$$

and the voltages at detectors $\mathrm{B}$ and $\mathrm{D}$ are

$$
V_{\mathrm{B}, \mathrm{D}} \propto \frac{1}{2}\left|\mathbf{E}_{1,2} \cdot(-\hat{x}+\hat{y})\right|^{2} .
$$

The differential amplifiers in each leg subtract the bias terms inherent in measuring intensity. The re- sulting inphase and quadrature voltages appearing at the outputs of the differential amplifiers are

$$
\begin{aligned}
& V_{I}=V_{A}-V_{B}=2 a_{e} a_{m} \cos \left(\phi_{e}-\phi_{m}\right), \\
& V_{Q}=V_{C}-V_{D}=2 a_{e} a_{m} \sin \left(\phi_{e}-\phi_{m}\right) .
\end{aligned}
$$

As long as $a_{e}$ and $\phi_{e}$ remain stable, this quadrature detector will measure both the inphase and the quadrature components of the TM field. If $a_{e}$ is also somehow either modulated or noisy, the addition to the detector of a third leg with a polarizer positioned to pass the TE field and block the TM field normalizes it. This leg will simply measure $a_{e}$, so that it may be divided out of $V_{I}$ and $V_{Q}$. There is no simple way to also account for variation in the phase of the TE field.

\section{Experimental Verification}

To investigate the sensitivity of the polarization quadrature measurement, we measured the polarization response for two samples and compared the measurements with numerical diffraction calculations. The first sample we used was a DVD, generously loaned to us by the Sony Corporation. The disc consists of an aluminum layer covered with $0.6 \mathrm{~mm}$ of polycarbonate. Depressions, representing the digital data, are formed in circular tracks in the aluminum layer. The depressions are nominally $0.11 \mu \mathrm{m}$ deep and $\sim 0.25-0.3 \mu \mathrm{m}$ wide (radial direction). The length (track direction) of the depressions varies from 0.4 to $2.128 \mu \mathrm{m}$ and depends on the recorded data. Therefore the depressions are generally much longer than they are wide. The tracks are spaced a constant $0.74 \mu \mathrm{m}$ apart. ${ }^{3}$ The actual pit dimensions of the disc used in our measurements were not measured and might differ from the nominal values given above. We will refer to the case in which the electric field is parallel to the tracks as the TE polarization.

The second sample was supplied by the Rochester Photonics Corporation (RPC). It consisted of 1- $\mu \mathrm{m}$ surface gratings fabricated in photoresist with a rastered laser spot. Variation of the intensity of the laser and special development of the resist enabled the RPC to fabricate sinusoidal gratings of constant period and varying depth. After receiving the sample, we evaporated $\sim 500 \AA$ of aluminum on the sample to form reflective gratings. As usual, we call the case in which the incident electric field is parallel to the grooves that form the grating the TE polarization.

The experimental setup is shown in Fig. 4. As explained in Section 3, we require that the incident spot be composed of equal components of TE- and TM-polarized light and the laser be aligned appropriately. The NA of the objective lens is 0.7 ; thus it forms an $\sim 1-\mu \mathrm{m}$ spot on the sample. However, the objective is not corrected for polarization. Since the deleterious polarization effects caused by an uncorrected objective increase with the radius of the exit pupil, we limited the detected light to the center of the exit pupil. Closing the size of the exit pupil was accomplished by imaging the pupil plane onto an iris. The iris was closed so that the effective numerical 


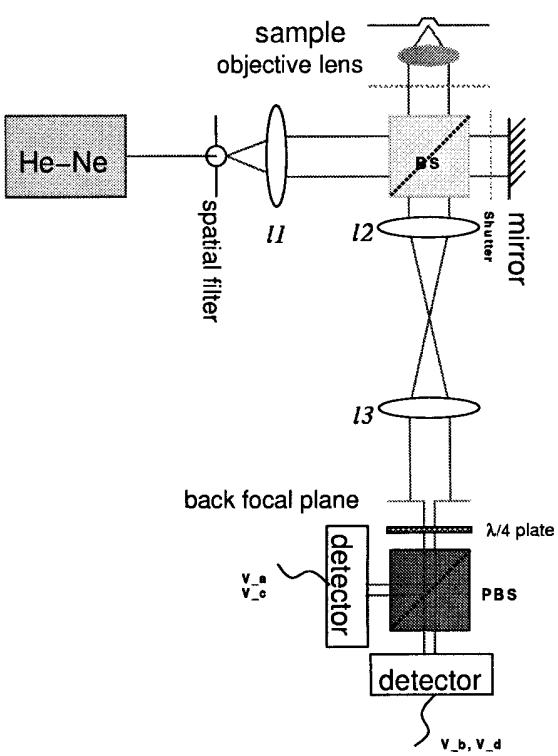

Fig. 4. Experimental setup. The He-Ne laser output polarization is set to be $45^{\circ}$ with respect to the grating direction of the sample. When the quarter-wave plate is set to $0^{\circ}$, the detector output $V_{a}$ and $V_{b}$, gives $V_{i}$. When the quarter-wave plate is rotated $45^{\circ}$, the output is $V_{q}$.

aperture of the detected light was $\sim 0.2$. Rather than use the architecture of Fig. 3, we simplified the detector by using only one polarizing beam splitter and a quarter-wave plate. The quarter-wave plate is rotated so that $V_{i}$ and $V_{q}$ are measured sequentially. The mirror leg of the nonpolarizing beam splitter is used to form an interferometer for focusing the incident spot. The shutter is closed during measurements.

The experimental results presented here will be compared with the two-dimensional, rigorous numerical calculations. We used the integral approach to solve the numerical diffraction problem at the interface between two homogeneous media. Our particular simulations were adapted to account for tightly focused spots and to eliminate the requirement for periodic structures. A more complete description of our numerical diffraction calculations can be found in Ref. 6.

The polarization in quadrature response for the DVD is shown in Fig. 5. In this graph the horizontal axis is $V_{i}$ and the vertical axis is $V_{q}$. The measurements were made starting in a region outside the outermost track, where a planar aluminumpolycarbonate interface is present. Measurements were then made sequentially as the disc was translated in 0.1- $\mu \mathrm{m}$ steps, until the illumination spot was well within the outermost track. Because of the random nature of the data pits, and because we cannot know at any specific time exactly where the illumination spot falls, we cannot make a definite correspondence between each measured data point and any specific theoretical point. Therefore we repeated the experiment at different locations, and we

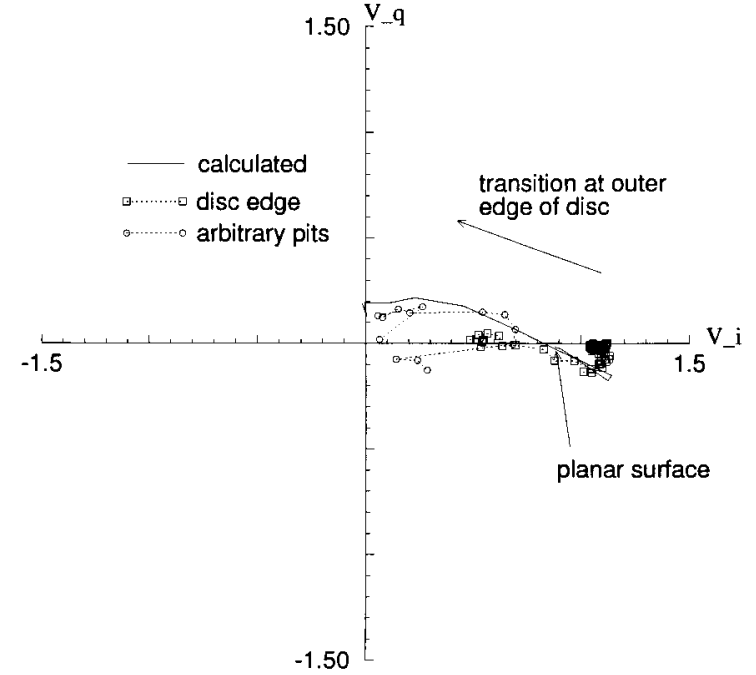

Fig. 5. Polarization in quadrature response for the DVD. The arrow points to the response for a planar aluminum-polycarbonate interface (planar surface), and the locus of points is the polarization response as the illumination spot moves from a region of flat aluminum to a region where recorded data tracks are present.

do not expect all the data points to lie close to the numerical calculations.

The agreement between the measured and the calculated data is good. In both cases the constellation for the transition from a planar interface to the presence of tracks starts with a small clockwise loop where the radius actually becomes larger. The increase in the amount of light that falls within the smaller numerical aperture (0.2) of the detected light means that some of the incident light from the edges of the aperture are diffracted into the center of the aperture. The constellation then moves above the real axis while moving in toward the origin.

Since $V_{i}$ and $V_{q}$ were measured sequentially and not simultaneously, we could check for inconsistencies in the data by considering the total power detected in each measurement. The total power measured is $\Sigma_{i}=V_{a}+V_{c}$ when the quarter-wave plate is set for $V_{i}$ and $\Sigma_{q}=V_{b}+V_{d}$ when the plate is set for $V_{q}$. These sums should be nearly equal since rotating the quarter-wave plate should not affect the total power, only the proportion transmitted and reflected by the polarizing beam splitter. Therefore we rejected measurements if the criterion

$$
A\left|\frac{\Sigma_{i}-\Sigma_{q}}{\Sigma_{i}+\Sigma_{q}}\right|<1
$$

was not satisfied. Generally, we set $A$ to a value between 20 and 50 . Some reasons why the criterion might not be met are laser fluctuations and vibrations or instabilities of the mounted disc.

We also had to account for the birefringence in the polycarbonate layer. The birefringence of the polycarbonate layer varies with the location on the disc and from disc to disc. The birefringence results from the injection-molding process, and the direction of the 


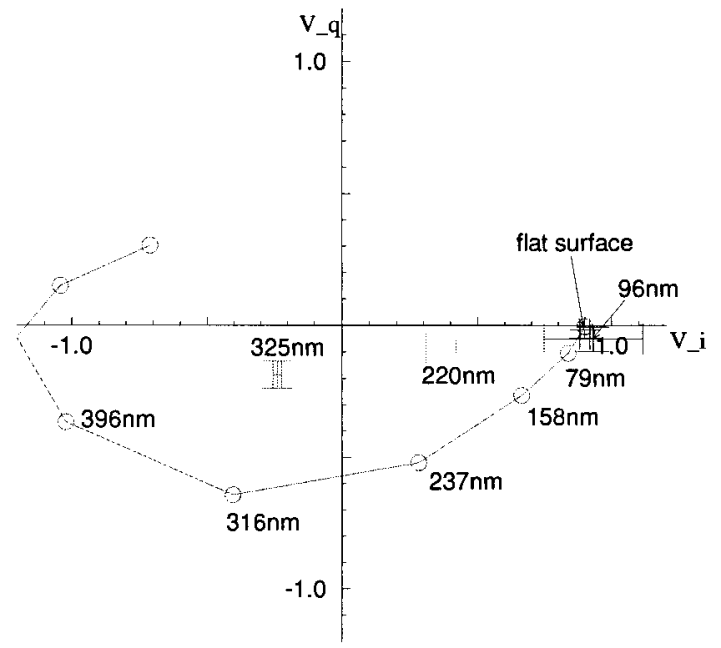

Fig. 6. Polarization in quadrature response for a $1-\mu \mathrm{m}$ sinusoidal aluminum grating. The arrows point to the response for a planar aluminum-air interface, and each data point, calculated or measured, is labeled with its corresponding grating depth.

principal axes depends on the flow direction and the thickness of the molding process. ${ }^{11}$ We measured the birefringence of the disc at a location outside the outermost track in the planar interface region, and we found that the principal axes of the birefringence were in the radial and the track directions. In this case, the effect is to simply rotate the measured polarization constellation. Our measurements indicated that amount of rotation resulting from the birefringence in the polycarbonate layer was between $21^{\circ}$ and $28^{\circ}$, and the data plotted in Fig. 5 account for this rotation.

To estimate the sensitivity of polarization measurement to small changes in the structure, we measured the polarization in quadrature responses for aluminum gratings with three different grating depths and a planar aluminum surface. The gratings were on a sample fabricated by RPC. Each grating had a period of $1 \mu \mathrm{m}$ and had a sinusoidal profile. The depth and the profile of each grating were verified with an atomic force microscope. The polarization measurements were performed with the same setup as for the DVD sample (see Fig. 4). Each measurement was made after the incident spot on a grating peak was aligned, and the measurements were repeated several times for each grating. The results are shown in Fig. 6, and the error bars reflect the standard deviation of the polarization measurements for each grating. Also shown in this figure are theoretical predictions for $1-\mu \mathrm{m}$ aluminum gratings of various depth. Although the amplitudes of the measured points are smaller than predicted, their succession in a clockwise direction agrees with the predicted trend. We suspect that the reduction in reflected power in the grating regions, compared with the flat surface, are due to nonuniform deposition of the aluminum coating in the grating valleys as well as scattering from increased surface roughness. Other contributors to measurement inaccuracy were inaccuracies in the alignment of the incident spot on a grating peak, laser fluctuations, and the vibrations inherent in using a fixed bench.

Agreement with theory is not necessary for a meaningful estimation of the sensitivity of PQM. The measured data are very encouraging as to the sensitivity of PQM to small changes in grating depth. For example, the distance between the measured constellation points corresponding to grating depths of 220 and $325 \mathrm{~nm}$ is about 4 times the width of the error bars for the first point and 10 times for the second. Thus an ability to measure the depth of an unknown grating with an accuracy of $10-25 \mathrm{~nm}$ is implied. With improved equipment and techniques, this sensitivity can be improved.

\section{Conclusion}

We have presented a new technique for the measurement of subwavelength structures. The physical principle involved is that the amplitude and phase of the diffraction from small structures are dependent on polarization. If a structure is known a priori to be a specific type of structure, for example, an aluminum line, then measuring its diffraction response to polarization will give information as to the line's width, height, etc. To measure the polarization response easily, including the relative polarization phase change, we constructed a detector system that uses multiple detectors and polarization optics.

Using a set of gratings with varying depth, we have experimentally shown that the polarization in quadrature measurement is very sensitive to small changes in depth. For this technique to be useful, we need to be able to predict what the polarization response would be for various types of structures. For this purpose we used rigorous electromagnetic numerical simulation. The accuracy of the simulation was verified when its results were compared to measurements with a DVD as a sample. Future research could involve investigating the suitability of this method for critical dimension metrology in microelectronic fabrication.

We are grateful to Seiji Kobayashi and Masanobu Yamamoto of the Sony Research Laboratories for supplying us with an example of a DVD. We are equally grateful to Dean Faklis of RPC for the sample containing gratings of various depths, and to Dan Wilson of the Jet Propulsion Laboratory for atomic force microscope measurements of the gratings. The research presented here was funded in part by the National Science Foundation Center for Neuromorphic Systems Engineering at the California Institute of Technology.

\section{References}

1. C. J. Raymond, M. R. Murnane, S. L. Prins, S. S. H. Naqvi, J. R. McNeil, and J. W. Hosch, "Multi-parameter CD measurements using scatterometry," in Metrology, Inspection, and Process Control for Microlithography X, S. K. Jones, ed., Proc. SPIE 2725, 698-709 (1996).

2. C. J. Raymond, S. S. H. Naqvi, and J. R. McNeil, "Scatterometry for CD measurements of etched structures," in Metrology, 
Inspection, and Process Control for Microlithography X, S. K. Jones, ed., Proc. SPIE 2725, 720-728 (1996).

3. Toshiba Digital Video Disc Brochure (Toshiba Corporation, 1995).

4. J. G. Dil and B. A. Jacobs, "Apparent size of reflecting polygonal obstacles of the order of one wavelength," J. Opt. Soc. Am. 69, 950-960 (1979).

5. Y. Kok and N. C. Gallagher, "Relative phases of electromagnetic waves diffracted by a perfectly conducting rectangulargrooved grating," J. Opt. Soc. Am. A 5, 65-73 (1988).

6. D. S. Marx and D. Psaltis, "Optical diffraction of focused spots and subwavelength structures," J. Opt. Soc. Am. A 14, 12681278 (1997).
7. J. Pasman, "Vector theory of diffraction," in Principles of $\mathrm{Op}$ tical Disc Systems (Hilger, London, 1985), Chap. 3.

8. H. Haidner, J. T. Sheridan, J. Schwider, and N. Streibl, "Design of a blazed grating consisting of metallic subwavelength binary grooves," Opt. Commun. 98, 5-10 (1993).

9. G. Sirat and D. Psaltis, "Conoscopic holography," Opt. Lett. 10, 4-6 (1985).

10. G. Sirat and D. Psaltis, "Conoscopic holograms," Opt. Commun. 65, 243-249 (1988).

11. J. P. Wu and J. L. White, "Study of birefringence character of injection and compression-molded polycarbonate and its interpretation,” Polym. Eng. Sci. 31, 652-660 (1991). 